\title{
Sur la stabilité des pentes et des pics
}

\author{
About the stability of slopes and peaks
}

\author{
Pierre DUFFAUT ${ }^{*}$
}

Rev. Franç. Géotech. n 63, pp. 57.64 (avril 1993)

\section{Résumé}

L'étude des versants instables et celle des bâtiments exceptionnels pourrait s'appuyer sur celle des versants stables de grande hauteur et raideur. Les données rassemblées sur des pentes des Alpes françaises et suisses, et sur quelques pentes de Norvège et de Nouvelle-Zélande montrent bien que la pente décroît quand la hauteur croît; elles montrent aussi que les pics et arêtes isolés dépassent de très loin les barrages. Assurément l'eau souterraine y est d'autant moins dangereuse que ces édifices naturels sont plus élancés. II n'y a pas de théorie satisfaisante sur la stabilité des versants extrêmes, et la confiance qu'on leur porte tient seulement à la rareté des mouvements de masse qu'on y constate.

\section{Abstract}

Studies for unstable slopes and for exceptional buildings could be founded on the knowledge of slopes stable in spite of their great height and steepness. Slope data gathered from French and Swiss Alps, and a few from Norway and New Zealand, show that the slope angle decreases when the height increases. Also that isolated peaks and ridges overpass by far the highest towers and dams. Certainly groundwater is the less dangerous inside the sharpest summits. There is no satisfactory theory about the stability of extreme slopes; we only rely on them owing to the scarcity of their failures.

\footnotetext{
- 130, rue de Rennes, 75006 Paris.
} 


\section{INTRODUCTION}

L'empirisme de l'ingénieur se base sur des essais plus ou moins réussis ; mais à l'origine il a dû mettre à profit les exemples proposés par la nature. Aujourd'hui encore ces exemples gardent toute leur valeur, surtout lorsqu'ils dépassent largement la pratique courante; c'est le cas des reliefs de hauteur et de raideur exceptionnelles, comme des cavernes naturelles de portée exceptionnelle. Ce qui est nouveau, c'est que le rationalisme du même ingénieur l'oblige à justifier ce qu'il observe avant de prétendre l'imiter.

Ainsi existe-t-il d'immenses salles souterraines naturelles, stables depuis des millénaires (GILLI, 1984); il a fallu attendre la patinoire olympique de Gjövik en Norvège (en fin de construction pour les prochains jeux d'hiver à Lillehammer) pour oser une salle souterraine de $62 \mathrm{~m}$ de portée, très loin au-delà de tous les précédents en génie civil, mais 5 ou 6 fois moins que la plus grande grotte connue (Nasib Lubang Bagus, Malaisie, $400 \mathrm{~m}$ de portée environ).

De même les pyramides naturelles des reliefs les plus hardis sont-elles sans commune mesure avec les constructions humaines; pour tirer parti de ces exemples, encore faut-il les connaître; peu d'études sont consacrées aux pentes "stables ".

L'étude présentée ici concerne exclusivement des pentes « extrêmes », tant par la hauteur que par la raideur. La démarche s'inspire de celle suivie à l'instigation de R. Thérond dans la comparaison entre les grottes naturelles de portée exceptionnelle et les cavernes des ingénieurs (GILLI, 1984; DUFFAUT et al., 1986). Comme pour les cavernes, elle confirme le rôle de l'eau souterraine, en outre elle conduit à séparer le comportement des pentes de celui des pics isolés (DUFFAUT, 1992), ce qui justifie le titre choisi.

\section{PRÉLIMINAIRES}

Comme ingénieur géologue à Electricité de France, lau. teur s'est trouvé constamment confronté à l'instabilité des pentes. En effet, il y a dans presque toutes les vallées de montagne des ouvrages d'EDF susceptibles de souffrir de ces mouvements. Plus grave encore, ils peuvent être soupçonnés de les avoir provoqués, puisque ces ouvrages apportent ou retiennent de l'eau; ainsi s'est-il convaincu que tout versant est un barrage (DUFFAUT et LOUIS, 1972).

En 1968, lauteur a constaté avec surprise que les exploitants de mines à ciel ouvert cherchaient par tâtonnements la «pente stable limite » qu'ils pourraient ensuite prolonger quelle que soit la profondeur, démarche qui négligeait l'eau (rare autour de certaines mines), mais surtout démarche contraire au théorème de Galilée sur l'impossibilité des géants (1). Don COATES (et al., 1963) paraît le premier à avoir mis, pour les mines à ciel ouvert, l'accent sur la cohésion comme on le sait au moins depuis Coulomb, la pente limite dépend de sa hauteur (et réciproquement); Coates propose le type de graphe repris ci-dessous pour séparer des talus de mines stables et instables.
Au Bureau de recherches géologiques et minières à partir de 1977, l'auteur a eu accès à de nombreuses études et publications, en particulier sur les volcans, les terrils et les barrages en remblais (notamment les digues à stériles), d'où cette conclusion que les volcans sont des terrils (DUFFAUT, 1987). Le glissement sous-marin du delta du Var (pendant le chantier de l'aéroport de Nice) a bien montré que, pour les reliefs naturels aussi, il faut séparer les pentes en déblai et en remblai.

\section{DONNÉES EXPLOITÉES}

L'étude est limitée à des pentes dont les mesures sont accessibles sur les cartes (2), à l'exclusion de toute mesure sur le terrain. Les meilleures cartes sont les cartes de Suisse aux $1 / 25000$ et $1 / 50000$, les cartes françaises ont été utilisées aussi et localement des cartes de Norvège et de Nouvelle-Zélande, malgré des échelles moins adaptées à ces mesures; les hauteurs dépassent toutes $500 \mathrm{~m}$, pour minimiser l'influence des erreurs de lecture des distances.

L'étude est limitée aux montagnes granitiques, pour éviter les conséquences trop évidentes des structures internes des roches stratifiées ou métamorphiques. Une grande quantité de mesures vient des massifs alpins, Mont-Blanc et Suisse. Il s'agit surtout de contreforts ou d'éperons au flanc des massifs, mais aussi de pics isolés et d'arêtes. Deux pics ont été plus précisément considérés, le Cervin en Suisse, le Stetinden en Norvège (visité en mai 1991), deux arêtes (3), la Meije en France, et le Mitre Peak en Nouvelle-Zélande (visité en février 1992). Toutes ces régions ont subi les glaciations quaternaires et restent proches des glaciers actuels.

- Le massif du Mont-Blanc s'allonge entre deux profondes vallées parallèles, avec des pentes dissymétriques, plus raides mais un peu moins hautes sur le versant italien :

- sur l'Italie: plus forte dénivelée $\quad 3080 \mathrm{~m}$ pente $33^{\circ}$, - sur la France: $\quad 3700 \mathrm{~m} \quad 25^{\circ}$, - et à l'aiguille Verte: $\quad 3000 \mathrm{~m} \quad 31^{\circ}$; mais les éperons et pics marginaux sont plus raides: - sur l'Italie: aig. Noire de Peuterey $2220 \mathrm{~m} \quad 43^{\circ}$ mont Noir de Peuterey $\quad 1200 \mathrm{~m} \quad 50^{\circ}$,

- sur la France: aig. du Midi (rocher) $\quad 1040 \mathrm{~m} \quad 53^{\circ}$, face supérieure de l'aig. du Plan

$650 \mathrm{~m}$

$62^{\circ}$

(1) Cette considération simpliste de la pente se perpétue dans la pratique des cartes de pentes, lorsque la hauteur et l'eau souterraine sont négligées.

(2) L'exploitation d'images satellites doit permettre de simplifier le travail présenté ici et de l'étendre à des régions peu cartogra. phiées.

(3) Arête est pris ici dans deux sens différents : arêtes d'un sommet puramidal (intersections de ses faces); et crêtes à multiples pointes de hauteur comparable, qui n'ont que deux faces, et qu'il vaudrait mieux appeler "râteaux ", à l'image de quelques sommets de la Vanoise et de l'Oisans. 
et les raideurs extrêmes sont observées sur des faces et promontoires donnant sur les glaciers internes:

- Grand Dru, sur la Mer de Glace

- éperon Walker (Grandes Jorasses)

- pointe Croux (aig. Verte)

- Grand Dru

sur la Charpoua au sud

$2000 \mathrm{~m}$

$42^{\circ}$

$1200 \mathrm{~m}$

$660 \mathrm{~m}$

$61^{\circ}$

$64^{\circ}$,

$920 \mathrm{~m}$

$59^{\circ}$,

- Grand Dru

sur la Charpoua au sud-est

$500 \mathrm{~m}$

$64^{\circ}$.

Ces exemples montrent bien que la pente diminue lorsque la hauteur augmente (et aussi que les récits d'alpinistes exagèrent volontiers lesdites pentes).

Le massif ne montre pas de sommet aussi isolé que le Cervin, mais il présente deux arêtes remarquables:

- les Grandes Jorasses: face SE face NW (déjà citée)

- lensemble Droites-Courtes: face NE face SW

$2500 \mathrm{~m}$ $1200 \mathrm{~m}$ $41^{\circ}$, $61^{\circ}$,

\section{$1200 \mathrm{~m} 51$ à $56^{\circ}$,} $700 \mathrm{~m} \quad 50^{\circ}$.

Les Alpes suisses sont plus riches en sommets isolés, dont le Cervin (ou Matterhorn, $4477 \mathrm{~m}$ ) est le plus remarquable exemple.

- Le Cervin (fig. 1) est une pyramide effilée à quatre faces, dominant de $1000 \mathrm{~m}$ les cols qui l'entourent sur la crête frontière entre Suisse et Italie, de 2500 et $2800 \mathrm{~m}$ les villages de Breuil au sud (Italie) et de Zermatt au nord (Suisse). Il a une pente moyenne de $60^{\circ}$

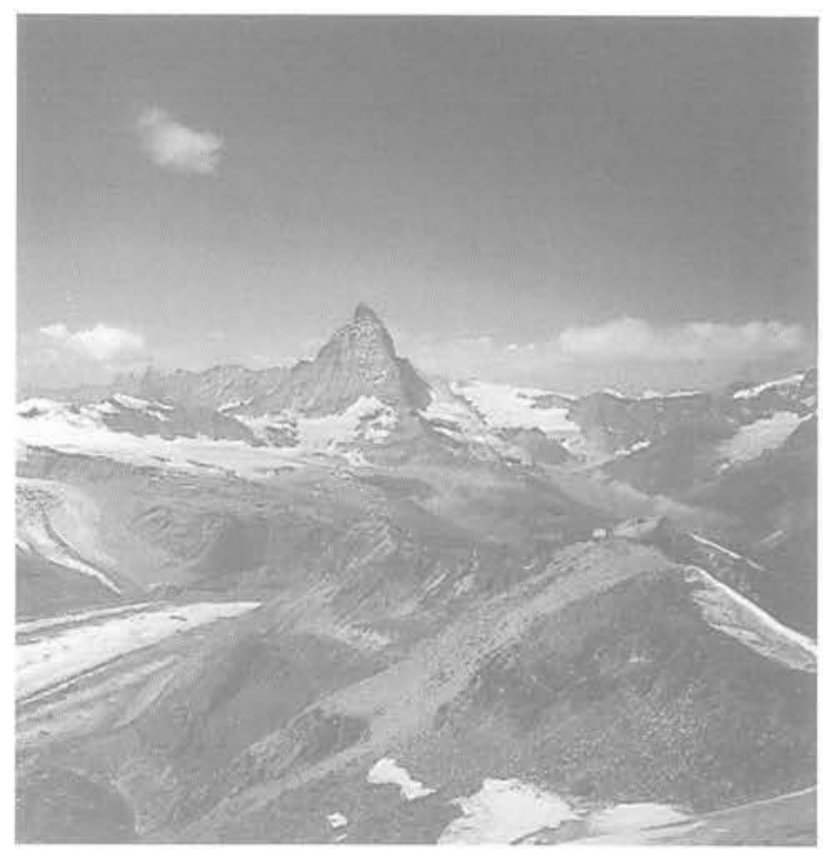

Fig. 1. - Le Cervin, vu de I'ENE (du Stockhorn); la face nord à droite, l'arête de Furggen à gauche; le seul 4000 proche, la dent d'Hérens, est cachée derrière le Cervin (photo de l'auteur).

Fig. 1. - Matterhorn, from ENE (Stockhorn); north face on right. Furggengrat on left: the only 4000 nearby. Hérens peak, is hidden behind Matterhorn. sur $1000 \mathrm{~m}$ pour l'ensemble de ses faces, de $38^{\circ}$ sur $1000 \mathrm{~m}$ pour l'ensemble de ses arêtes:

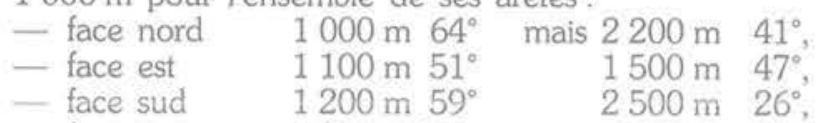

- face ouest $1350 \mathrm{~m} 56^{\circ}$.

- arête ENE

$1500 \mathrm{~m} 36^{\circ}$

(a. du Hornli, voie normale),

- arête SE

$1150 \mathrm{~m} 48^{\circ}$

(a. de Furggen

sur Furggjoch),

- arête WSW

$1000 \mathrm{~m} \mathrm{26} 6^{\circ}$ (sur col Tournanche)

- arête NW

$2000 \mathrm{~m} 40^{\circ} \quad$ (arête de Zmutt)

- Le Weisshorn $(4505 \mathrm{~m})$ est une pyramide à trois faces très régulières, mais avec une arête de faible hauteur et pente au NNW $\left(400 \mathrm{~m}\right.$ à $\left.14^{\circ}\right)$; sa face ouest est haute de $1300 \mathrm{~m}$ à $50^{\circ}$; il domine à l'est la vallée de la Viège de $3100 \mathrm{~m}$ à $33^{\circ}$.

- La Dent blanche (4356 m) a quatre faces, avec une arête sud de $500 \mathrm{~m}$ à $27^{\circ}$ seulement; sa face NE est haute de $900 \mathrm{~m}$ à $57^{\circ}$. Au voisinage le Zinalrothorn $(4221 \mathrm{~m})$ a des faces de hauteur plus modestes mais plus raides: face est, $680 \mathrm{~m}$ à $68^{\circ}$ (record enregistré dans les Alpes) ; face ouest, $410 \mathrm{~m}$ à $54^{\circ}$.

- Plus au nord, le Finsteraarhorn (4274 m), dans le massif de l'Aar, est une aiguille isolée sur une longue arête ; sa face NE est haute de $1050 \mathrm{~m}$ à $63^{\circ}$, comparable à la face nord du Cervin.

- En Norvège, près de Narvik, le Stetinden (Gudens ambolt, Enclume du Dieu, en raison de son curieux sommet tronqué, cf. fig. 2), s'élève d'un seul jet à $1392 \mathrm{~m}$ au bord du Stefjord. C'est une pyramide à trois faces dont la partie supérieure est plus effilée que le Cervin sur au moins $700 \mathrm{~m}$ de haut (mais seulement dans une direction, il se dresse à l'extrémité d'une sorte d'arête) :

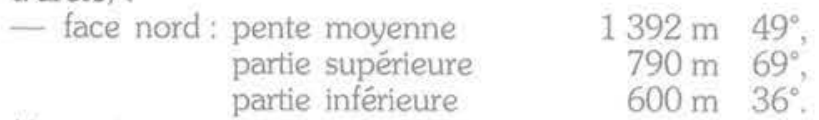

(il ne s'agit pas d'éboulis, mais de roche en place, en dalles parallèles au versant, découpées par la décompression):

- face ouest: régulièrement concave $1200 \mathrm{~m} 40^{\circ}$

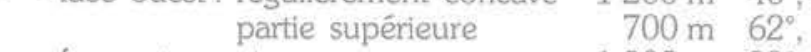

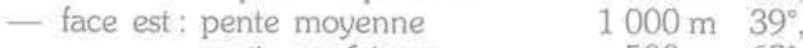
partie supérieure $\quad 500 \mathrm{~m} 62^{\circ}$.

Les environs montrent beaucoup de parois raides quoique de hauteur inférieure à $1500 \mathrm{~m}$, et beaucoup de crêtes très dissymétriques, une face en pente douce ou même un plateau étant opposé à une face raide: - maximum de $68^{\circ}$ sur $500 \mathrm{~m}$ (Presttinden, $1336 \mathrm{~m}$ ) - pentes de 45 à $50^{\circ}$ sur $1000 \mathrm{~m}$ à $1200 \mathrm{~m}$, $40^{\circ}$ sur $1450 \mathrm{~m}$.

En raison de l'échelle et du figuré des courbes de niveau, ces données sont moins précises que dans les Alpes, mais en revanche il faut souligner combien la concavité de certains versants est extraordinairement régulière, parfois sur des kilomètres de longueur (Hangbakktinden en rive droite du Skjomenfjord, pente moyenne $40^{\circ}$ sur $1400 \mathrm{~m}$, avec une tangente verticale au sommet, sur environ $5 \mathrm{~km}$ ).

- Les arêtes de la Meije (3987 m), dans le massif de l'Oisans (fig. 3), sont célèbres par leur face sud très 


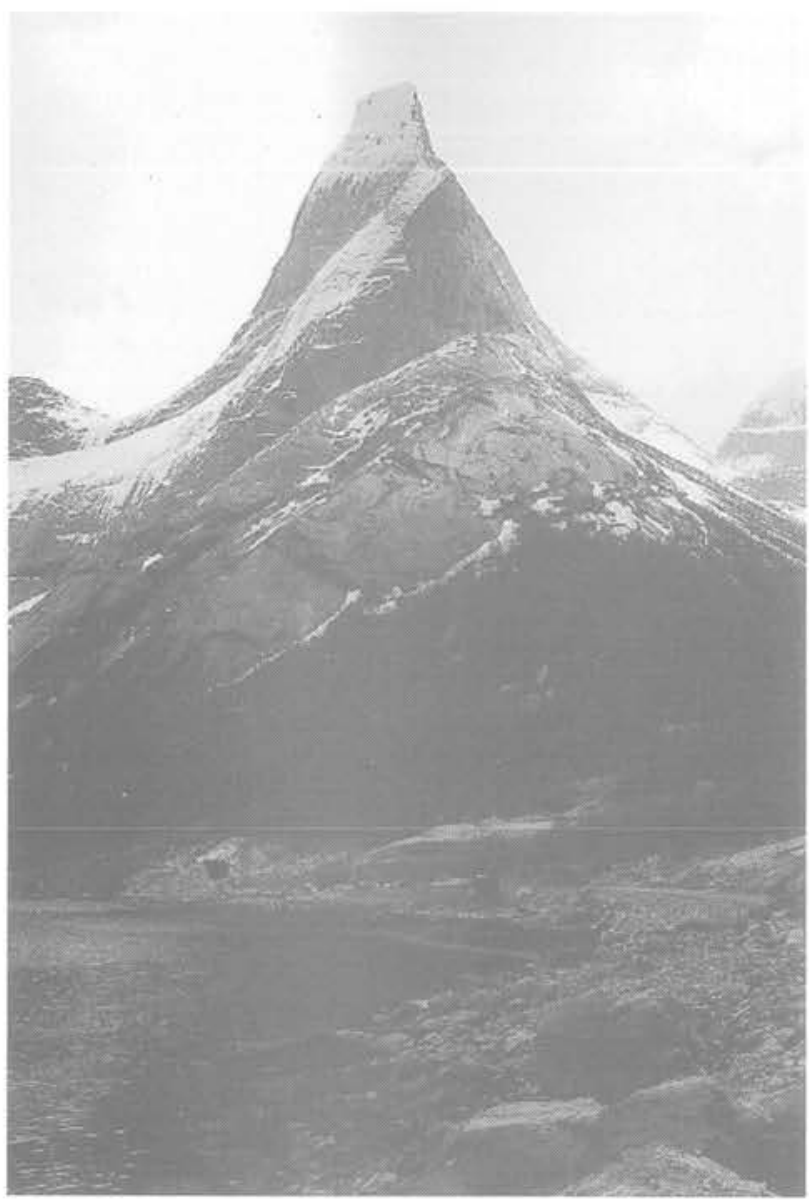

Fig. 2, - Le Stetinden, vu du NW (de la route de Kjöpvik, en construction) ; la face nord vue de profil à gauche, la face ouest à droite; l'escalade attire des grimpeurs de tout premier plan (photo de l'auteur). Fig. 2. - Stetinden, from NW (the road to Kjöpvik. then under construction): north face in profile on left, west face on right. first rank climbers come there from worldwide.

raide, opposée à une face nord glaciaire en pente mo. dérée:

- face nord: jusqu'au torrent partie supérieure

- face sud: facette NNW

$$
\begin{array}{rr}
2500 \mathrm{~m} & 31^{\circ}, \\
2000 \mathrm{~m} & 41^{\circ}, \\
700 \mathrm{~m} & 60^{\circ}, \\
700 \mathrm{~m} & 66^{\circ},
\end{array}
$$

(murs verticaux alternant avec des replats glaciaires).

- En Nouvelle-Zélande, le Mitre Peak (fig. 4) est l'attraction touristique de lîle du Sud, Milford Sound, Fjordland. Il domine les eaux du fjord de $1694 \mathrm{~m}$. Il s'agit d'une longue arête, de près de $3 \mathrm{~km}$, qui culmine d'ailleurs un peu au-delà du sommet nommé; contrairement aux deux exemples précédents, la pente diminue au voisinage du sommet. Le massif au sud du Milford Sound est creusé de profondes vallées séparées par de minces arêtes, par exemple le sommet nommé Terror Peak. La carte de la rive droite du fjord n'étant pas disponible, il n'a pas été possible de mesurer les pentes très raides que l'on $y$ voit (peut-être $75^{\circ}$ sur un millier de mètres, fig. 5):

- Mitre: face fjord

face derrière

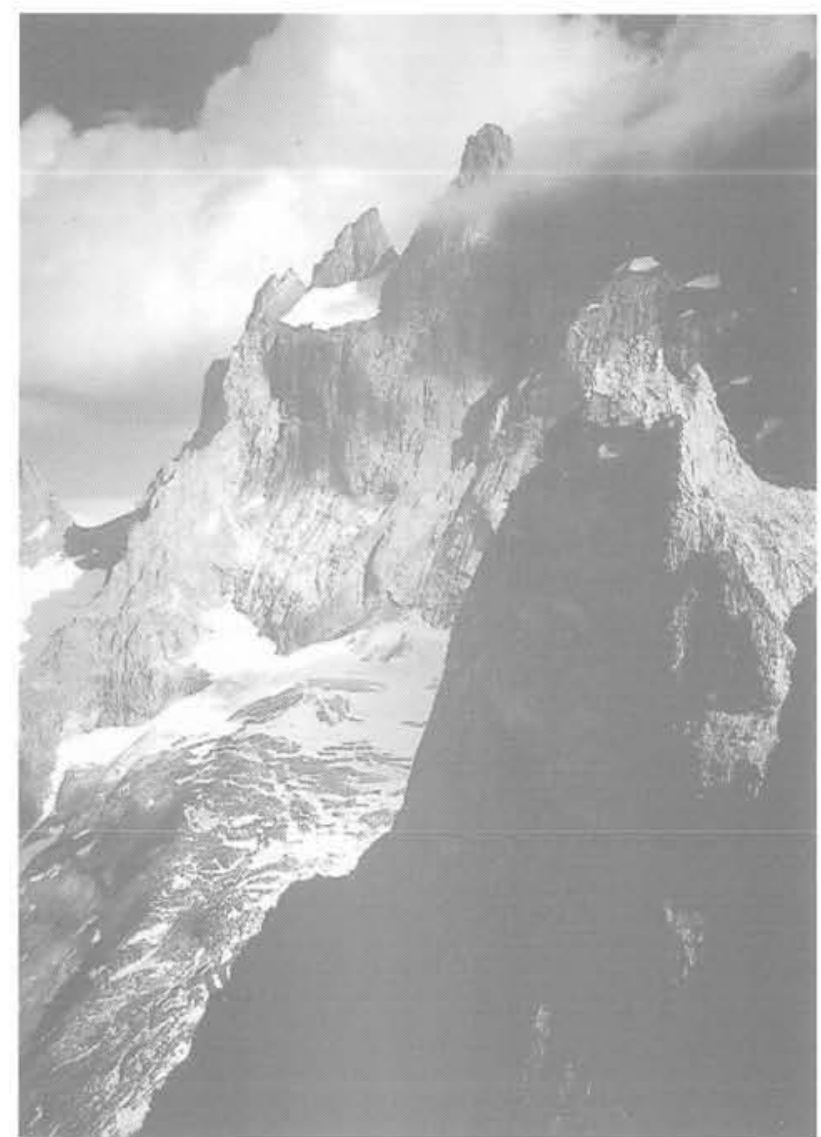

Fig. 3. - La Meije, vue du SSE; la face sud des arêtes de la Meije est vue en biais, de la Brèche

et du "promontoire " qui porte le refuge du même nom, au sommet du Grand Pic, point culminant,

au centre le Glacier carré, la muraille s'étend à droite en perdant de la hauteur (photo de l'auteur).

Fig. 3. - La Meije, from SSE; south face of the ridge. seen at an angle; from left to right «la Breche \& pass.

the "Glacier Carré " over the "Promontoire » hut. and the main summit "Grand Pic »: the height of the wall decreases from left to right.

$-2^{\circ}$ sommet: face fjord

- Terror: face NE face derrière

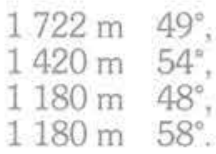

\section{OBSERVATIONS}

Souvent la pente augmente de façon monotone de la base au sommet avec une tangente verticale (Cervin, mais aussi beaucoup de versants de vallées glaciaires, notamment en Norvège ou au sud-est de la NouvelleZélande), à la façon de la pente maximale théorique en milieu cohérent (les surplombs sont négligés ici). D'où les sommets effilés lorsque de tels versants se recoupent.

Beaucoup de pics célèbres par leur aspect effilé sont plutôt des contreforts, au flanc d'un sommet plus massif, ainsi l'aiguille du Dru devant l'aiguille Verte (massif du 


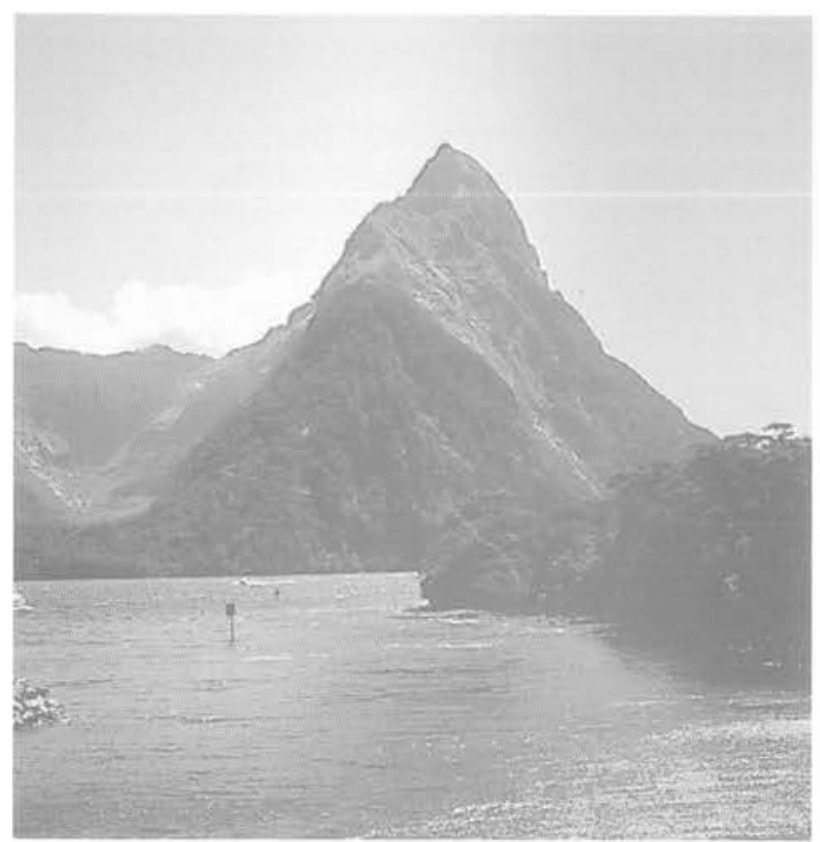

Fig. 4. - Le Mitre Peak, vu de l'ESE, de la piste d'atterrissage de Milford Sound, presque dans l'axe de l'arête (photo de l'auteur).

Fig. 4. - Mitre Peak, from ESE (Milford Sound runway). nearly in the axis of the ridge.

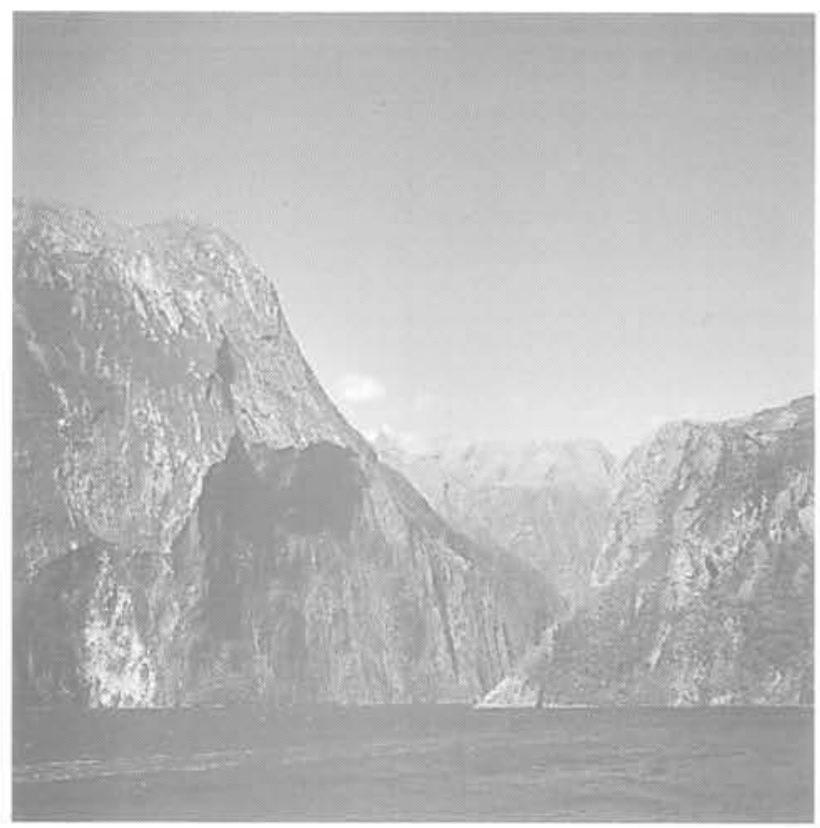

Fig. 5. - Milford Sound, paroi de $1000 \mathrm{~m}$ de haut en face du Mitre Peak, avec un surplomb impressionnant; l'abondance de la végétation (il s'agit bien de grands arbres) est due à un climat très arrosé (photo de l'auteur).

Fig. 5: - Milford Sound. $1000 \mathrm{~m}$ high wall opposite to Mitre Peak, with an impressive overhang

Mont-Blanc), ou l'extrémité d'une arête, ainsi la Dibona (massif de l'Oisans). Même la prestigieuse aiguille Verte n'est que l'extrémité (surélevée) de l'arête Droites. Courtes.
Les arêtes des sommets isolés sont aussi des contreforts, comme on en voit aux angles de certains clochers; mais les sommets en dièdres (arêtes subhorizontales) sont souvent dépourvus de contreforts perpendiculaires significatifs et les versants rectilignes signalés en Norvège n'en ont pas du tout.

Les pentes des versants de vallées glaciaires approchent de la verticale (par exemple Milford Sound) et sont souvent composites, une partie témoignant par les « moutonnements » polis et striés de l'action superficielle d'usinage du glacier, une autre des arrachements et écroulements contemporains de l'écoulement et de la fonte du glacier (fig. 5).

\section{INTERPRÉTATION}

Les résultats ont été résumés d'abord (fig. 6) par un nuage de 56 points sur un graphe pente-hauteur (un point de Mitre Peak venant combler un "gap " du graphe publié par DUFFAUT, 1992, en s'inscrivant audelâ des parois du Cervin et du Stetinden). La courbe enveloppe des versants extrêmes peut s'exprimer sous forme hyperbolique:

$$
1 / \theta=0,01+610^{-6} \mathrm{H}
$$

(avec $\mathrm{H}$ hauteur en mètres, $\theta$ angle en degrés).

Les chiffres donnés au paragraphe 4 sont répartis sur cinq graphes de détail (fig. 7 à 11); à l'exception des pics suisses (fig. 8) une limite rectiligne parait suffisamment représentative, faute peut-être de points au-dessous de $500 \mathrm{~m}$.

Les versants jugés instables (ou connus comme tels) seraient très loin du bord droit du nuage; en général leur instabilité a des causes structurales volontairement écartées ici.

Les points les plus à droite appartiennent aux pics et arêtes isolés : trois causes peuvent justifier cette stabilité : - les contraintes verticales de poids propre, inférieures en moyenne à $\gamma \mathrm{H}$ (de $1 / 3$ à $1 / 2 \gamma \mathrm{H}$ pour des pyramides ou des cônes, ou des arêtes à 2 faces, proches de $\gamma \mathrm{H}$ pour les mêmes volumes tronqués);

- les contraintes d'écoulement de l'eau souterraine, puisque la nappe ne peut monter aussi haut, à conditions hydrologiques comparables dans des volumes élancés que sous des massifs épais;

- le gel, qui scelle les parois des aiguilles et limite les infiltrations (et la pente qui prévient l'accumulation de neige); au-dessus d'une certaine altitude, l'isotherme zéro empêche l'existence d'eau liquide; cette isotherme est d'autant plus basse que l'aiguille est plus effilée.

Il n'apparait pas de différence significative entre les pics isolés et les arêtes, ce qui peut s'expliquer par une certaine anisotropie du terrain, favorable à l'arête, et masquant l'augmentation des contraintes.

Les versants proches de l'enveloppe sont évidemment dépouvus de surfaces de discontinuité inclinées (les hautes faces verticales sont l'apanage des calcaires stratifiés en bancs subhorizontaux, à diaclases subverticales, par exemple dans les Dolomites). 


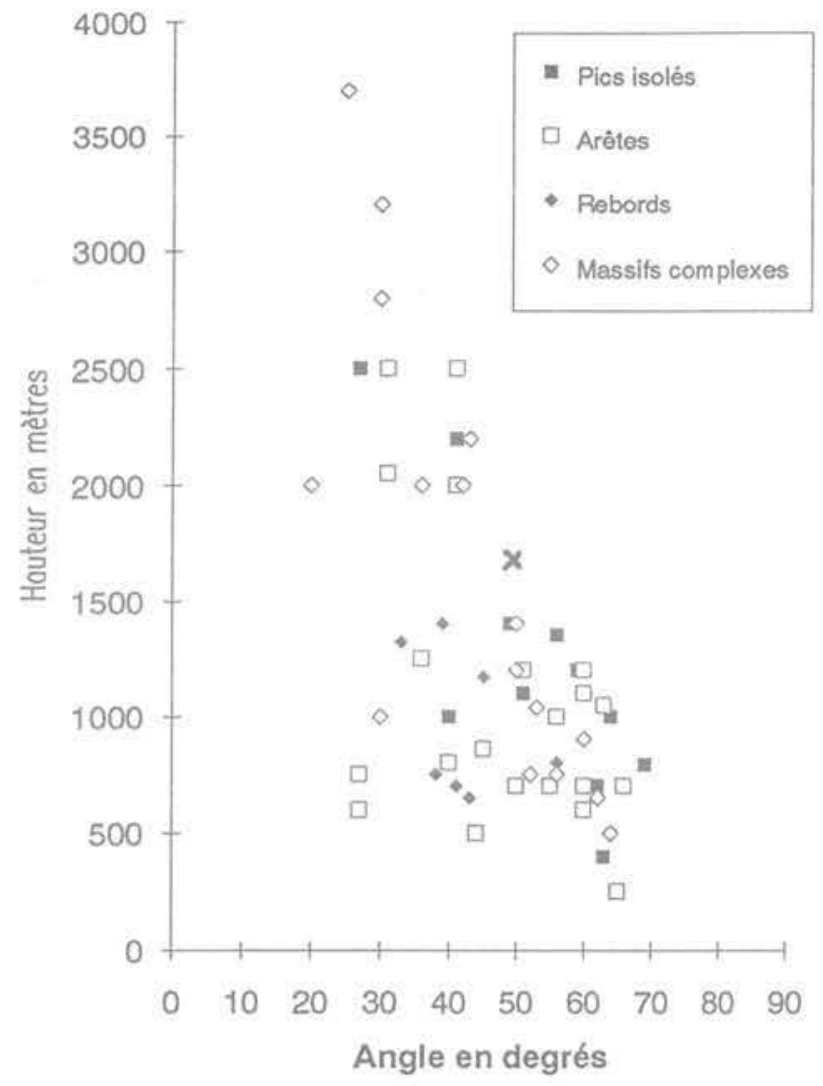

Fig. 6. - Graphe synthétique (DUFFAUT, 1992), la croix ajoutée à $1700 \mathrm{~m}$ vient du Mitre Peak et complète

la "courbe enveloppe 》 des versants extrêmes.

Fig. 6. - Synthetic slope-height graph (DUFFAUT.

1992): the cross at $1700 \mathrm{~m}$ is added from Mitre Peak

and complements the envelope of extreme slopes.

La géomorphologie n'a pas définitivement expliqué la genése de ces pentes et de ces pics ou arêtes; la jeunesse relative des érosions glaciaires peut justifier qu'on ne trouve nulle part ailleurs de telles pentes; leur évolution vers des pentes de plus en plus faibles est sans aucun doute inéluctable, ce n'est plus de la mécanique, mais de la géologie; leur durée de vie n'en est pas moins très grande par rapport aux constructions humaines (comme pour les cavernes extrêmes).

\section{COMPARAISONS AVEC LES OUVRAGES DE L'INGÉNIEUR}

Tumulus et pyramides témoignent de l'audace des anciens bâtisseurs (KÉRISEL, 1991) y compris la fameuse tour de Babel (ziggourat Etemenanki à Babylone, $91 \mathrm{~m}$ ) ; la pyramide n'est-elle pas selon ALAIN (1931, Leçons sur l'architecture, cité par Kérisel) le secret modèle de tous les édifices? Pour n'en citer que trois:

- Khéops, $147 \mathrm{~m}$ (aujourd'hui 138), pente $52^{\circ}$;

- le Mont-Saint-Michel, $148 \mathrm{~m}$ (avec la partie construite) ;

- l'Obélisque de Washington, $169 \mathrm{~m}$.
A côté des pyramides, l'Egypte propose d'ailleurs aussi le modèle des dièdres ou arêtes, sous la forme des "pylônes " érigés de part et d'autre des portes. Beaucoup moins hauts, ils sont aussi beaucoup plus raides et se rapprochent donc des murs verticaux.

Barrages : pour les barrages poids en béton, la somme des fruits va de 0,7 à 0,9$)$; si la face amont est verticale la pente est donc à l'aval 48 à $55^{\circ}$, plus faible quand la hauteur croît. Le record est le barrage suisse de Grande Dixence 283 m, $48^{\circ}$.

Les barrages en remblais ont des pentes beaucoup plus faibles, le record est le barrage russe de Rogun $325 \mathrm{~m}$.

L'ingénieur est donc loin d'approcher les hauteurs de certains édifices naturels. Aux débuts de la Terre Armée, Vidal avait fixé à $10000 \mathrm{~m}$ la hauteur limite des murs verticaux que ce procédé permettrait; on n'a pas dé. passé quelques décamètres!

C'est seulement grâce à l'acier et à la précontrainte que les constructions ont atteint et dépassé les records des barrages : tour Eiffel, $330 \mathrm{~m}$, gratte-ciel, tours de télévision, etc.

L'étude des vallées étroites est un complément à celle des pics élancés. Elle peut trouver application dans le domaine des barrages. La recherche des versants les plus raides est au service des projets de conduites forcées et de téléfériques.

\section{RETOUR SUR LA STABILITÉ DES VERSANTS}

Les massifs étudiés sont loin d'être les plus hauts du monde; mais l'Himalaya et la cordillère des Andes n'ont pas de bonnes cartes; tout reste à faire dans ces massifs, avec l'espoir qu'une hauteur plus grande mette mieux en valeur les résultats.

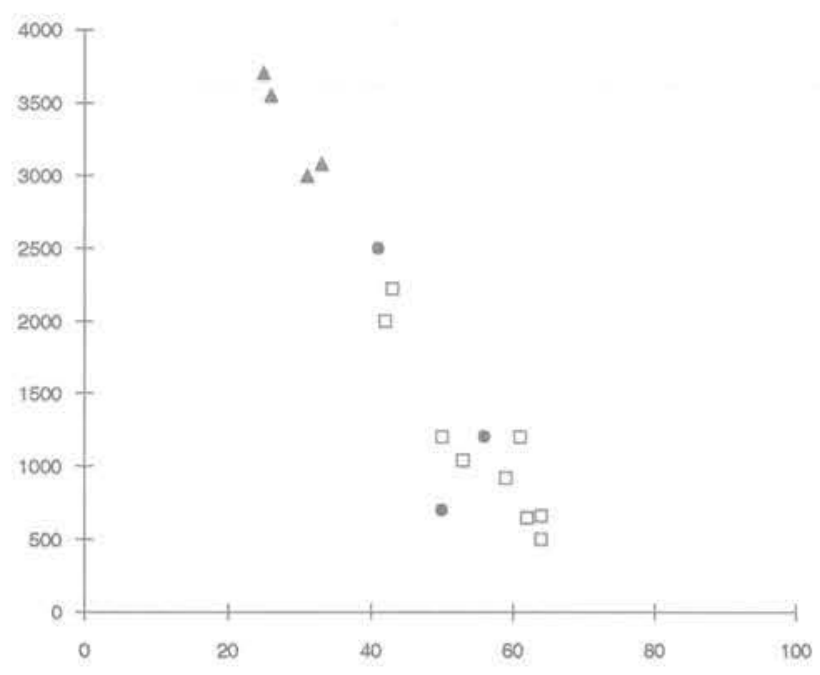

Fig. 7. - Graphe pente-hauteur pour 16 pentes du massif du Mont-Blanc, individualisant les promontoires (carrés), les arêtes (ronds) et les pentes moyennes composites (triangles),

Fig. 7. - Slope-height graph for 16 slopes from Mont-Blanc massif: squares for spurs or promontories. circles for ridges, triangles for composite slopes. 


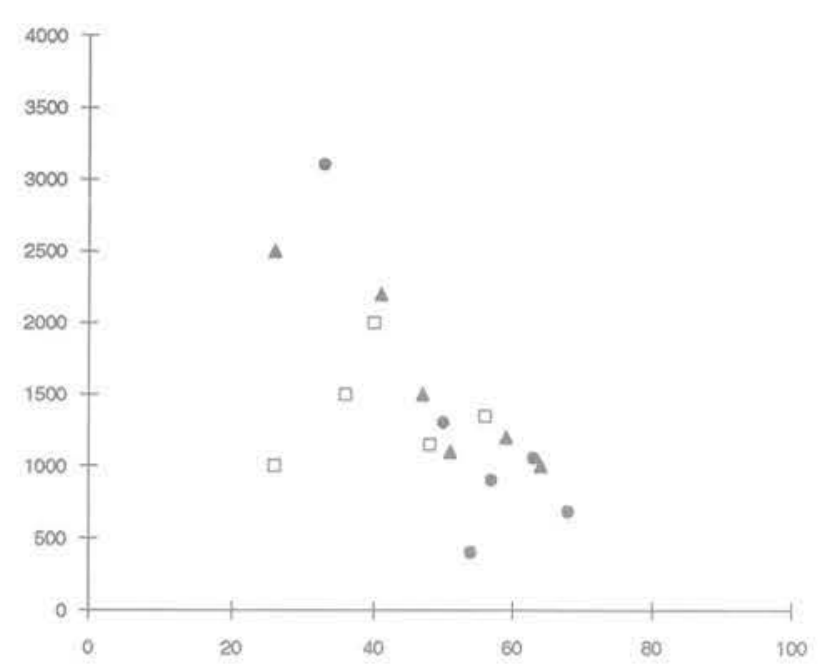

Fig. 8, - Graphe pente-hauteur pour 17 pentes de sommets suisses, dont les faces (triangles) et les arêtes (carrés) du Cervin:

les ronds proviennent d'autres sommets isolés.

Fig. 8. - Slope-height graph for 17 slopes from Swiss peaks; triangles for Matterhorn faces, squares for Matterhorn edges, circles for other isolated peaks.

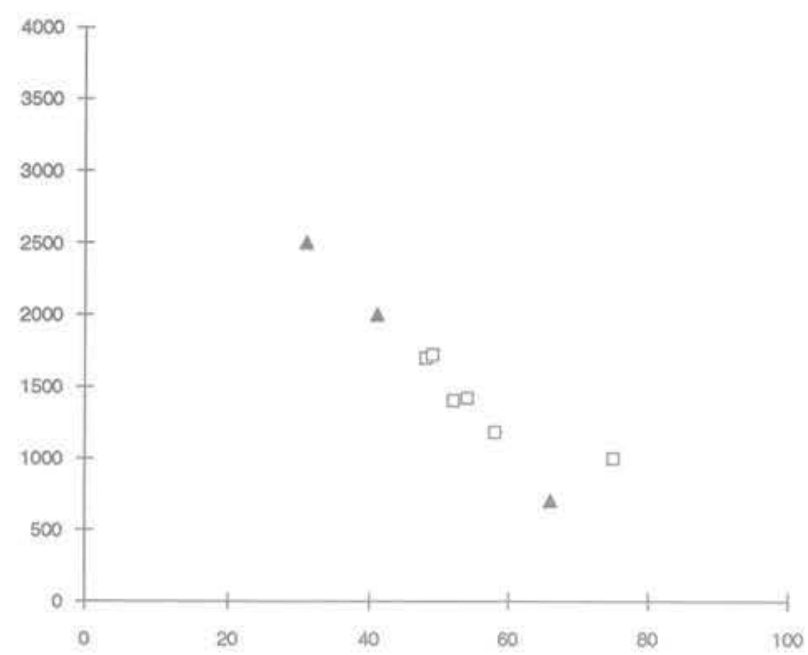

Fig. 10. - Graphe pente-hauteur pour des pics allongés en arêtes, la Meije (3 triangles) et le Mitre Peak (6 carrés); le carré isolé à droite vient d'une paroi au nord du Milford Sound.

Fig. 10. - Slope-height graph for ridges: la Meije (3 triangles) and Mitre Peak (6 squares). the isolated square is from the wall on fig. 5.

Comme pour les talus artificiels, en déblai ou en remblai, il y a lieu de classer les pentes naturelles, par exemple de la manière suivante:

- reliefs construits :

- isolés : édifices volcaniques,

- marginaux : éboulis, cônes alluviaux,

- mixtes: moraines, dunes, etc. ;

- reliefs soulevés : escarpements de failles;

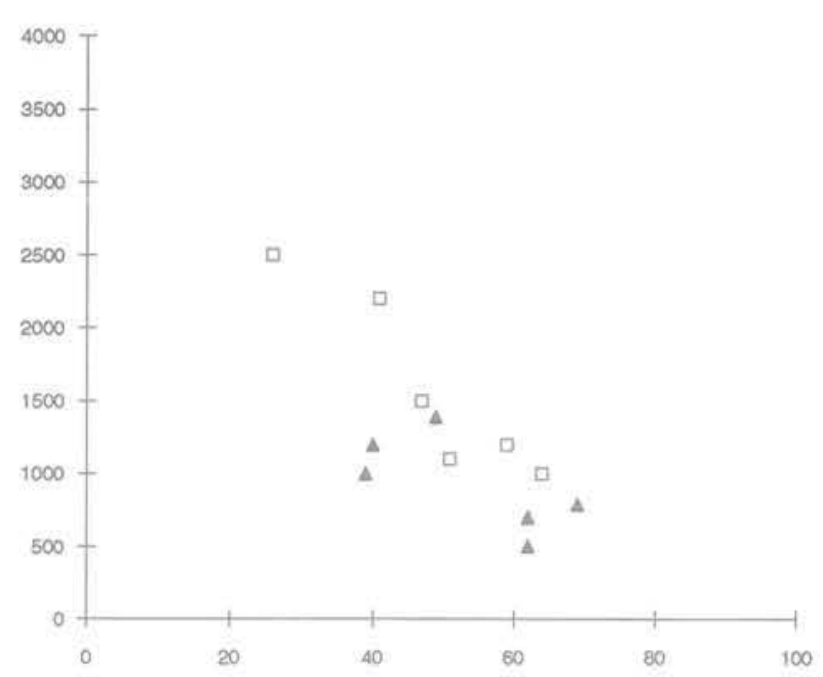

Fig. 9. - Graphe pente-hauteur pour les faces de deux pics effilés, le Cervin (carrés) et le Stetinden (triangles) (12 mesures).

Fig. 9. - Slope-height graph for the faces of 2 slender peaks. Matterhorn (squares) and Stetinden (triangles).

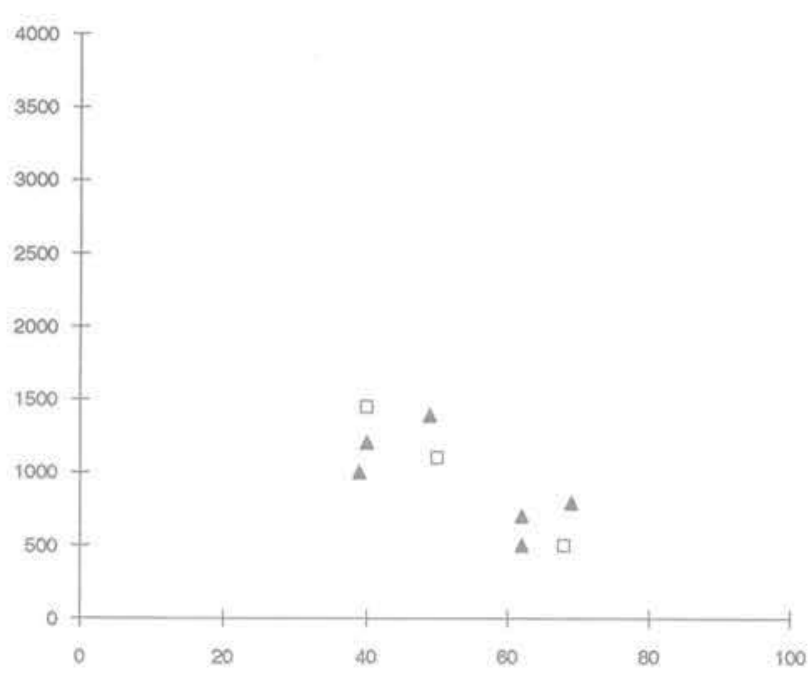

Fig. 11. - Graphe pente-hauteur pour 9 versants des environs de Narvik, dont le Stetinden (triangles).

Fig. 11. - Slope-height graph for 9 slopes around Narvik, including Stetinden (triangles).

- reliefs excavés:

- isolés : pics et arêtes, nunataks, etc.,

- marginaux : vallées, niches et cirques, falaises en bord de mer, etc.;

- reliefs résiduels (où l'altération prime le creusement).

Enfin, l'état actuel de tout versant doit être considéré comme transitoire, exactement comme l'état de santé d'un individu vivant. L'immobilité de la nature minérale n'est qu'une illusion d'optique, due à la faible durée relative de la vie humaine. "Tout coule » a écrit Démocrite, c'est une conséquence inéluctable de la pesanteur. 
Le métier de l'ingénieur géologue, confronté à l'instabilité des pentes est comparable à celui du médecin ; il doit :

- reconnaître la maladie ;

- juguler son évolution;

- tirer de chaque cas des conclusions de portée gé. nérale.

\section{CONCLUSION}

Les pentes extrêmes existent, et méritent beaucoup d'at. tention.

Les pentes extrêmes posent deux défis à l'ingénieur: d'abord comprendre comment et pourquoi cette stabilité hors du commun? ensuite, s'il y a lieu, comment et jusqu'où peut-on imiter la nature? Toutefois, le caractère exceptionnel des exemples présentés interdit une trop grande confiance.

Il faut admettre qu'il n'y a pas de pentes stables à long terme, mais le long terme des géologues laisse une marge confortable aux ingénieurs (la fatigue et les altérations sont au nombre des agents de l'érosion); en sens inverse tous les habitants des vallées profondes sont menacés d'ensevelissement "prématuré », avec un risque statistique faible (par comparaison avec d'autres risques couramment acceptés), mais jamais nul.

La richesse du sujet est loin d'être épuisée; de nombreuses études sont à mettre en œuvre dans des directions qui ne sont pas encore toutes recensées aujourd'hui ; des applications nouvelles apparaitront.
Robert THEROND a inspiré le démarrage de ces études, Knut-lvar SIMONSEN a initié l'auteur aux pentes des fjords norvégiens, Jean PÉRA a contribué aux idées présentées; qu'ils en soient vivement remerciés.

\section{RÉFÉRENCES}

COATES D. et al. (1963), Trans. AlME (réf. incomplète mais essentielle).

DUFFAUT P. (1987), Séisme et glissements de terrain au mont Ontake (Japon), (conf. du 21 janv. 1986). Ann. ITBTP, 457, février, pp. 47-69.

DUFFAUT P. (1992), Comparaison de quelques pentes extrêmes par la hauteur et la raideur. Int. Symp. on Landslides, Christchurch, David Bell editor, Balkema, pp. 65-70.

DUFFAUT P. et LOUIS C. (1972), L'eau souterraine et l'équilibre des pentes naturelles. $24^{\circ}$ Congr. Géol. Int., Montréal, Section 13, pp. 107-116, et Bull. BRGM, $2^{e}$ série, III, $n^{\circ} 4$, pp. 3-12.

DUFFAUT P., PIGUET J.P. et THÉROND R. (1986), A review of large permanent rock caverns in France. Int. Symp. on Large Rock Caverns, Helsinki, Kari Saari editor, Pergamon Press.

GILLI E. (1984), Recherches sur le creusement et la stabilité de grands volumes souterrains. Thèse, Université de Marseille.

KÉRISEL J. (1991), La Pyramide à travers les âges. Paris, Presses des Ponts et Chaussées. 